\title{
Immunomodulatory and neuroprotective mechanisms of Huangqi glycoprotein treatment in experimental autoimmune encephalomyelitis
}

\author{
Yanxia Xing ${ }^{1}$, Binyu Liu ${ }^{1}$, Yijin Zhao ${ }^{1}$, Lihong Zhang ${ }^{1}$, Rodolfo Thome ${ }^{2}$, Huiqing Xue ${ }^{3}$, Peijun Zhang ${ }^{1}$, Cungen Ma ${ }^{1}$ \\ ${ }^{1}$ Institute of Brain Science, Medical College, Shanxi Datong University, Datong, China, ${ }^{2}$ Department of Neurology, Thomas Jefferson \\ University, Philadelphia, Pennsylvania, United States, " “2011" Collaborative Innovation Center/Research Center of Neurobiology, \\ Shanxi University of Chinese Medicine, Taiyuan, China
}

\begin{abstract}
Previous studies have shown that Huangqi glycoprotein (HQGP) has an anti-inflammatory effect in vitro, and suppressed experimental autoimmune encephalomyelitis (EAE), an animal model of multiple sclerosis; however, the mechanism underlying its effect is largely unknown. In this manuscript we investigated the mechanisms by which HQGP protect mice from EAE. HQGP was extracted from Astragalus membranaceus and purified by anion-exchange and gel filtration chromatography. HQGP delayed disease onset, reduced disease severity and alleviated inflammation and demyelination in the central nervous system (CNS). Moreover, HQGP reduced the infiltration of pathogenic immune cells and increased the expression of microtubule-associated protein 2 (MAP-2) and neuronal nuclei (NeuN) in the CNS. HQGP treatment also reduced the expression of chemokines such as CCL2 and CCL5 and the production of tumor necrosis factor $\alpha$ (TNF- $\alpha$ ), interleukin (IL)-1B, IL-6, but increased the level of IL-10. These results demonstrate that HQGP suppressed EAE development by modulating the immune system and the infiltration of leukocytes to the CNS as well as promoting axon and neural repair.
\end{abstract}

Key words: Huangai glycoprotein, experimental autoimmune encephalomyelitis, immunocyte, chemokines, nerve repair.

\section{Introduction}

Multiple sclerosis (MS) is a chronic inflammatory demyelinating disease of the central nervous system (CNS) [9]. CD4 ${ }^{+} T$ cells, monocytes/macrophages, dendritic cells, B cells and other immune cells are involved in the pathophysiology of MS and its animal model experimental autoimmune encephalomyelitis (EAE) $[4,8,18]$. Immune cells release inflammatory mediators such as tumor necrosis factor (TNF), interleukins (IL), interferon (IFN) and chemokines that perpetuate inflammation in the CNS and lead to axon and neu- ronal damage. CC chemokines can induce inflammatory cells to migrate and accumulate into the CNS $[17,19,23,28]$, and their expression highly correlates with the severity of EAE and MS $[2,10]$. For example, CCL2 and CCL5 promote the adhesion of leukocytes to endothelial cells, which further prompts leukocyte activation, leading to demyelination and axon damage in MS patients [25,30]. Given that current MS treatments display certain disadvantages such as severe side effects and a high cost, new therapeutic approaches that address these issues will be of the utmost importance. 
Natural compounds and their derivatives have become an important source of new MS therapies $[7,14,20]$. The dry root of Astragalus membranaceus (commonly known as Huang Qi) is one of the most commonly used Chinese traditional medicines for its multitude of beneficial effects that include liver protection, anti-aging, anti-stress, and has been used to treat immune-related diseases [13,15,31]. In this study we investigated the protective effect of Huangqi glycoprotein (HQGP) on EAE. We found that HQGP could effectively improve the clinical symptoms of EAE, delay onset and regulate the immune function and its mechanisms of action rely on modulation of the immune system and neuroprotection.

\section{Material and methods \\ Mice}

Twenty 9-week-old female C57BL/6 mice with body weight of 18-22 g were purchased from Vital River Laboratory Animal Technology Co. Ltd. (Beijing, China), and kept at the Experimental Animal Center of Brain Science Institute of Shanxi Datong University. All experiments were conducted in accordance with the guidelines of the International Council for Laboratory Animal Science. The study was approved by the Ethics Committee of Shanxi Datong University, Datong, China (Grant No. 2017001).

\section{Huangqi glycoprotein}

HQGP was provided by the Experimental Center of Shanxi University of Chinese Medicine. The main chemical components of Astragalus membranaceus were Astragalus polysaccharides, saponins, flavonoids, amino acids and trace elements. Astragalus membranaceus was extracted by Tris- $\mathrm{HCl}$ buffer. The crude extract was purified by anion-exchange and gel filtration chromatography, then freeze-dried and identified as electrophoretically pure HQGP. The relative molecular weight of HQGP was $16.8 \mathrm{kDa}$. HQGP was dissolved in normal saline (NS) and diluted to $0.02 \mathrm{~g} / \mathrm{l}$ with deionized water for use.

\section{Experimental autoimmune encephalomyelitis induction and Huangqi glycoprotein treatment}

Mice were randomly divided into two groups: the vehicle-treated control group (NS) and HQGP treatment group (HQGP). Induction and assessment of EAE were performed according to our previous research [33].
Briefly, EAE was induced by subcutaneous immunization of the upper dorsal flanks with $300 \mu \mathrm{g}$ of myelin oligodendrocyte glycoprotein peptide 35-55 (MOG $\left.{ }_{35-55}\right)$ in complete Freund's adjuvant (Sigma, USA) supplemented with $3 \mathrm{mg} / \mathrm{ml}$ of Mycobacterium tuberculosis (BD Difco, USA) (400 $\mu \mathrm{g} /$ mice). Mice were then injected with $700 \mathrm{ng}$ of pertussis toxin (Alexis, USA) as immunopotentiator via the abdominal cavity on the day of immunization and again $48 \mathrm{~h}$ later. HQGP was injected intraperitoneally at $1 \mathrm{mg} / \mathrm{kg} /$ day on day 3 post-immunization (p.i.) until day 18 p.i. Mice from the NS group were injected with the same volume of vehicle.

\section{Clinical score}

The clinical development of EAE was assessed since the day of immunization. Changes in body weight were also recorded daily. Clinical assessment of EAE scores was evaluated daily using the international grading standard of 0-5 as instructed [32].

\section{Hematoxylin/eosin staining and Luxol fast blue staining}

On day 18 p.i., mice were perfused with normal saline and fixed with buffered paraformaldehyde (40 g/l) after anesthesia. The spinal cords (lower thoracic-lumbar) and brains of mice were removed and sliced $(7 \mu \mathrm{m})$. The pathological changes of CNS of mice were detected by hematoxylin/eosin ( $\mathrm{H} \& \mathrm{E})$ staining. Demyelination in the white matter of the spinal cord of mice was observed by Luxol fast blue (LFB) staining. The number of inflammatory cells in each whole spinal section as well as the ratio of demyelination area to the white matter area in mice were determined by Image Pro Plus 6.0.

\section{Immunofluorescence staining}

Slices were incubated with $3 \%$ bovine serum (Serotec, Bicester, UK) for 30 minutes at room temperature (RT) to block unspecific binding. The frozen sections were incubated with primary antibodies at $4^{\circ} \mathrm{C}$ overnight. Antibodies used in this study were: anti-CD11b (1 : 1000), anti-CD4 (1 : 1000), antiCD68 (1 : 1000; all from eBioscience), anti-CCL2 (1 : 500; Abcam, UK), anti-CCL5 (1 : 500; R\&D Systems Inc.), anti-MAP-2 (1 : 500; Millipore) and antiNeuN (1 : 500; Millipore). Then the sections were incubated with corresponding secondary antibodies labeled with Alexa Fluor 555/488 (1 : 1000; Cell Signaling) at RT for $2 \mathrm{~h}$. The number of positive cells in each section was counted by Image Pro Plus 6.0. 


\section{Detection of cytokines by enzyme-linked immunosorbent assay}

Spleens were collected from EAE mice at day 18 p.i. and prepared for single cell suspension. After lysis of red blood cells, the mononuclear cells (MNCs) were adjusted to $5 \times 10^{6} / \mathrm{ml}$. MNCs were stimulated with $\mathrm{MOG}_{35-55}(10 \mu \mathrm{g} / \mathrm{ml})$ for $72 \mathrm{~h}$ at $37^{\circ} \mathrm{C}$ in a humidified atmosphere containing $5 \% \mathrm{CO}_{2}$. The supernatant was collected and assayed for the detection of TNF- $\alpha$, IL-1 $\beta$, IL- 6 and IL-10 by enzyme-linked immunosorbent assay (ELISA) kits (R\&D Systems Inc.) following the manufacturer's instructions. The results are expressed as $\mathrm{pg} / \mathrm{ml}$.

\section{Statistical analysis}

All experimental values are expressed as mean \pm SD and analyzed by GraphPad Prism 5.0 software. Analysis between two groups were carried out by Student's $t$ test and values of $p<0.05$ were considered statistically significant.

\section{Results}

\section{Huangqi glycoprotein delays disease onset and reduces clinical severity of experimental autoimmune encephalomyelitis}

To investigate whether HQGP could suppress EAE development, we immunized $\mathrm{C} 57 \mathrm{BL} / 6$ mice to induce EAE and half of the mice were daily treated with HQGP. Mice receiving NS were used as controls. Our results show that mice from the NS group had a disease incidence of $100 \%$ and the onset was at day $8.60 \pm 0.84$ p.i. The mean maximum score was $3.50 \pm 0.67$. Conversely, HQGP treatment delayed disease onset $(11.60 \pm 1.78$ p.i.) and reduced the maximum clinical score $(1.60$ \pm 0.52 ). From day 8 p.i., the daily clinical score of NS-treated control EAE mice was higher than that of HQGP treated group (Fig. 1A). HQGP also relieved weight loss of mice (Fig. 1B).

\section{Huangqi glycoprotein modulates inflammation and alleviates demyelination in the central nervous system}

We then evaluated the inflammation in the CNS of EAE mice. Histological analysis revealed that mice from the NS group had an extensive infiltration of inflammatory cells into the white matter of the spinal cord and brain (Fig. 2). Conversely, HQGP-treated mice had a significant improvement of inflammation in the CNS compared with NS-treated mice (Fig. 2). Analysis of the demyelination in the spinal cords revealed that the area of demyelination in the HQGP treatment group decreased significantly compared with mice from the NS group (Fig. 3).
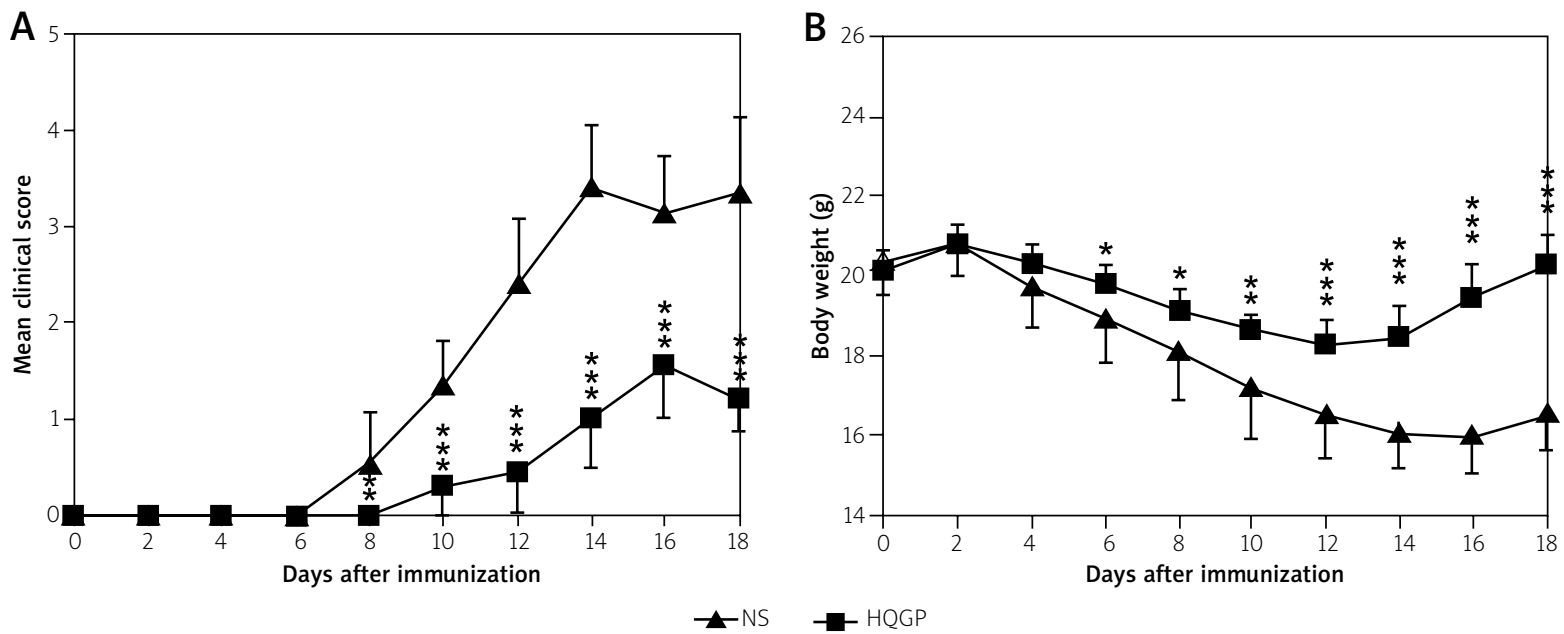

Fig. 1. Huangqi glycoprotein (HQGP) ameliorated the clinical course of EAE. C57BL/6 mice were injected i.p. with normal saline (NS) or HQGP (1 mg/kg/d), daily from day 3 p.i. (A) Clinical score and (B) Bodyweight were evaluated daily in a blinded fashion from days 0 to 18 p.i. Results are expressed as mean \pm SD ( $n=6$ each group). ${ }^{*} p<0.05,{ }^{* *} p<0.01,{ }^{* * *} p<0.001$. 

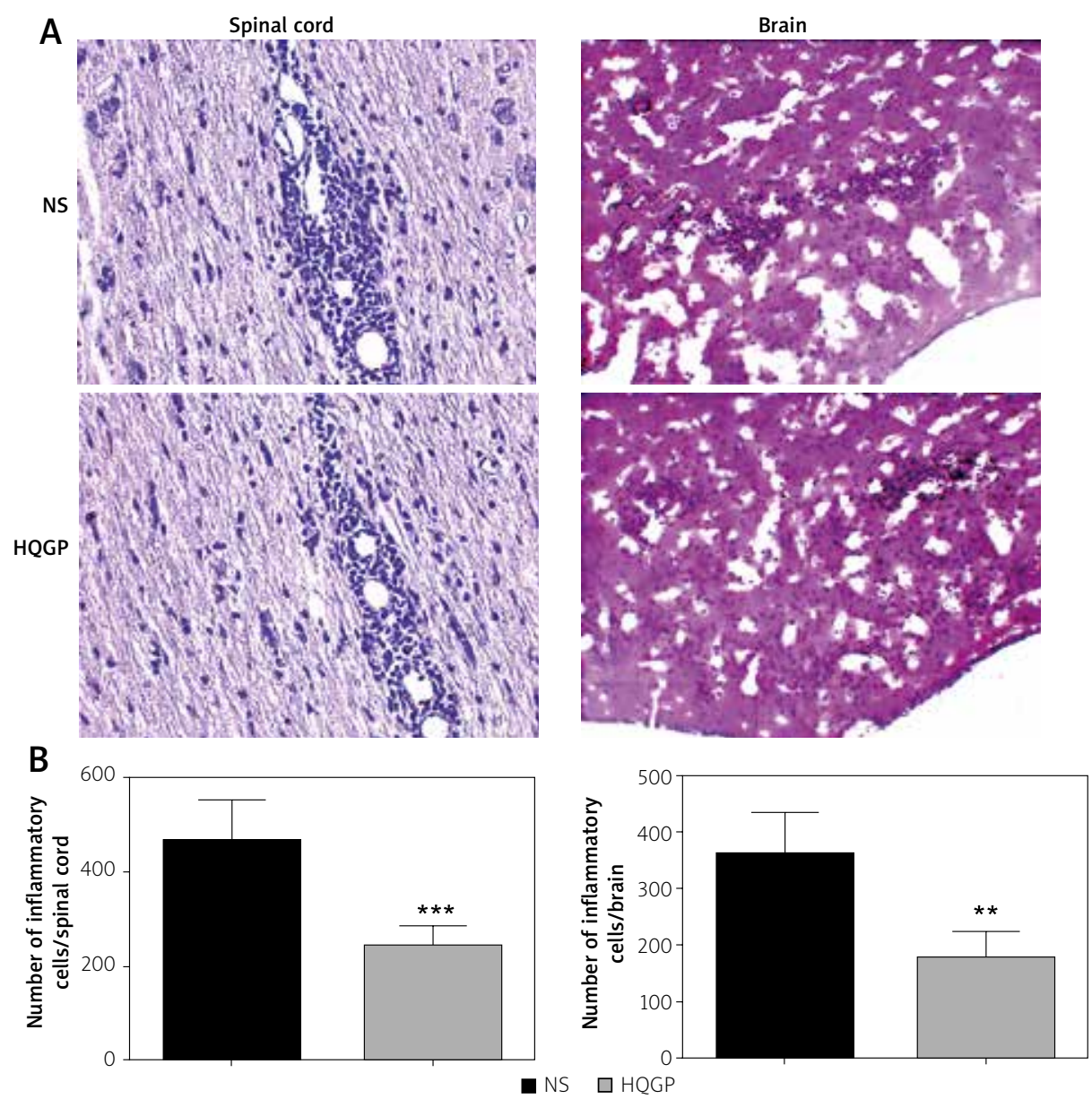

Fig. 2. Huangqi glycoprotein (HQGP) treatment reduced CNS infiltration. Mice described in Fig. 1 were dissected on day 18 p.i. and the brain and spinal cord were harvested. A) H\&E staining of the spinal cord and brain (H\&E, 400x). B) The number of inflammatory cells in the whole section of the spinal cord and brain was calculated separately by Image-Pro Plus 6.0 software. Results are expressed as mean \pm SD $(n=6$ each group). ${ }^{* *} p<0.01,{ }^{* * *} p<0.001$. One representative of three experiments with similar results is shown.
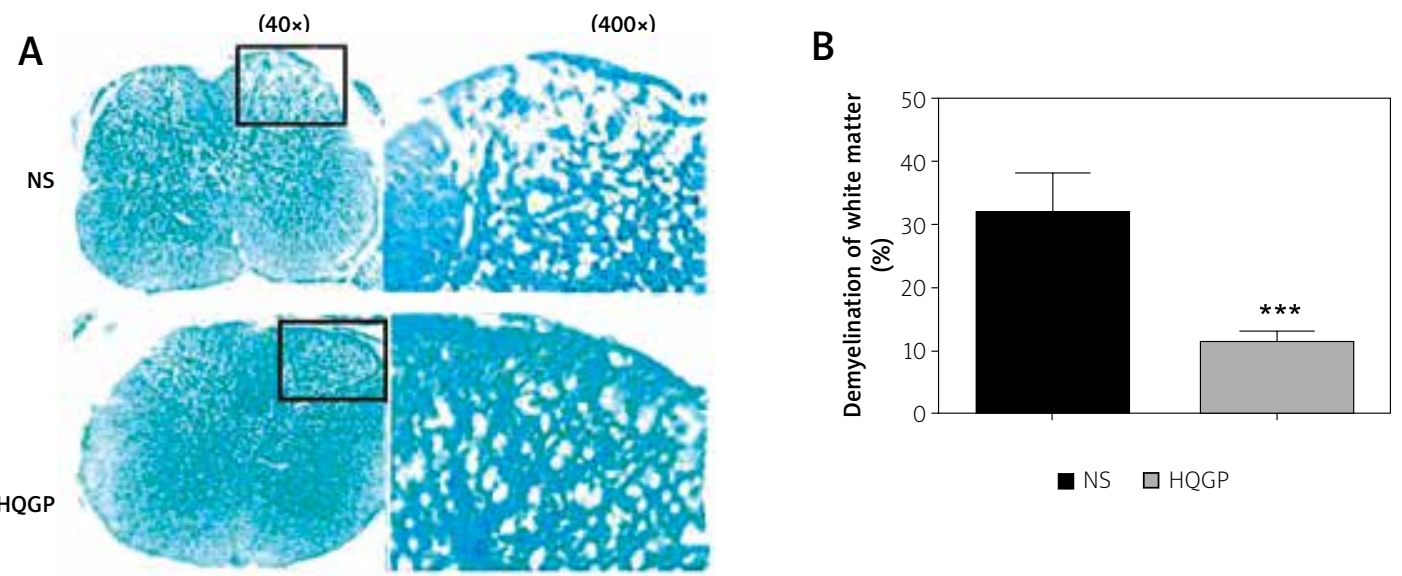

Fig. 3. Huangqi glycoprotein (HQGP) treatment reduced CNS demyelination. EAE mice were sacrificed on day 18 p.i., the spinal cord was harvested, and LFB staining was carried out for myelin evaluation. A) Representative digital images (40x and 400x). B) The pixel area (\%) of demyelination in white matter was calculated by Image-Pro Plus 6.0 software. Results are expressed as mean \pm SD ( $n=6$ each group). ${ }^{* * *} p<0.001$. One representative of three experiments with similar results is shown. 

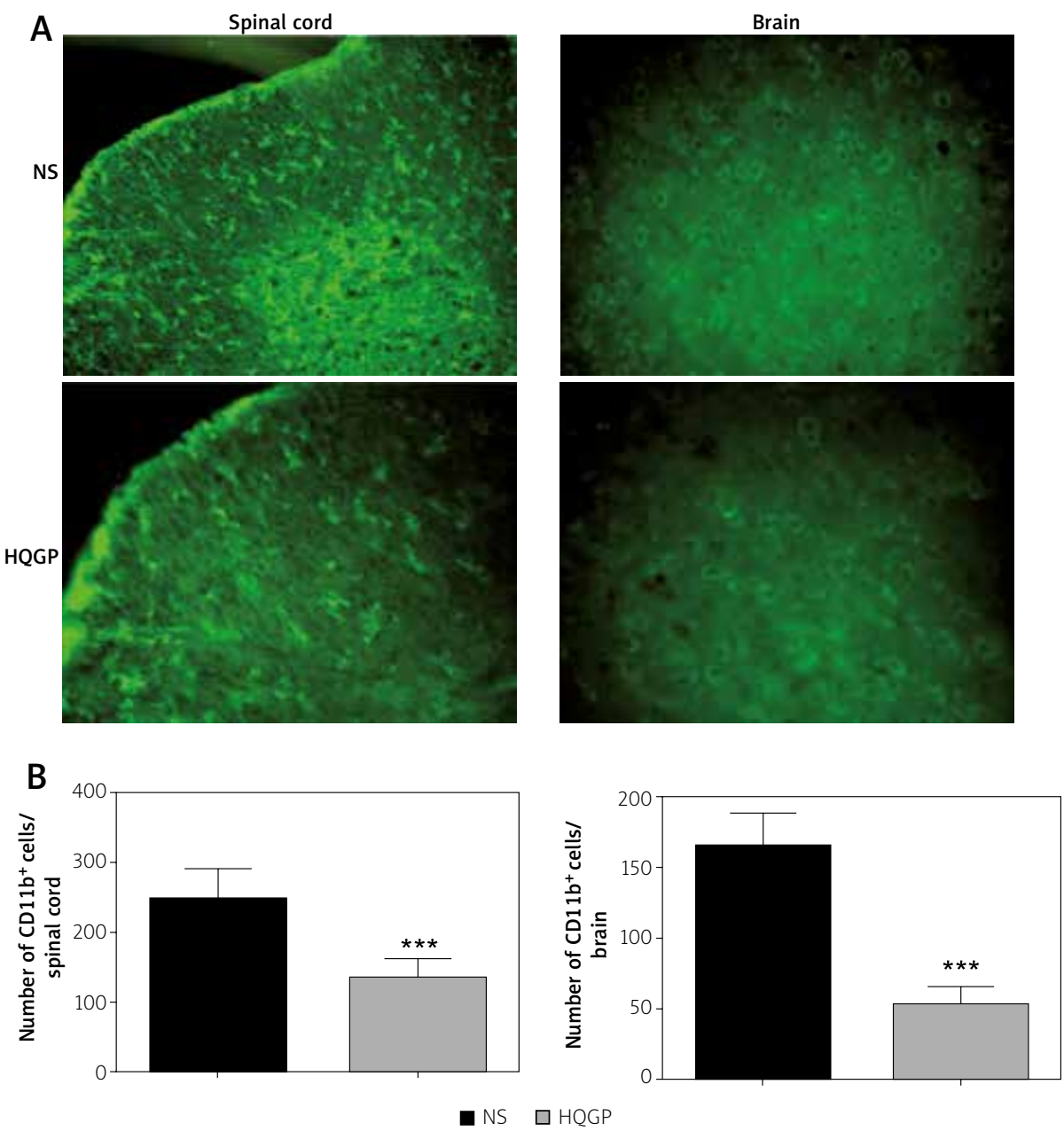

Fig. 4. Immunohistochemistry of $C D 11 b^{+}$monocytes in the CNS. The spinal cord and brain were harvested on day 18 p.i. and determined with anti-CD11b antibodies. A) Representative digital images (400x). B) The number of $C D 11 b^{+}$cells was calculated by Image-Pro Plus 6.0 software. Results are expressed as mean $\pm \mathrm{SD}$ ( $n=6$ each group). ${ }^{* *} p<0.001$. One representative of three experiments with similar results is shown.

\section{Huangqi glycoprotein reduces immune cell infiltration in the central nervous system of experimental autoimmune encephalomyelitis mice}

Characterization of the cells that infiltrated the CNS revealed that NS-treated mice had a high fre-

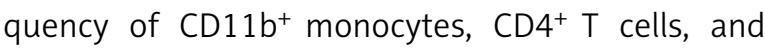
$\mathrm{CD}^{+} 8^{+}$macrophages as a result of CNS inflammation. Moreover, mice from the HQGP-treated group showed a significantly reduced frequency of these cells compared to mice from the NS group (Figs. 4-6).

\section{Huangqi glycoprotein inhibits the expression of $\mathrm{CCL} 2, \mathrm{CCL} 5$ and CCR5 in experimental autoimmune encephalomyelitis control mice}

Given that we observed a reduction in the cellular infiltration in the CNS of HQGP-treated mice, we analyze the production of CCL2 and CCL5 in the CNS by immunofluorescence as these chemokines play a major role in the CNS infiltration by leukocytes during EAE. We also analyzed the expression of CCR5, the receptor for CCL2 and CCL5, in the infil- 

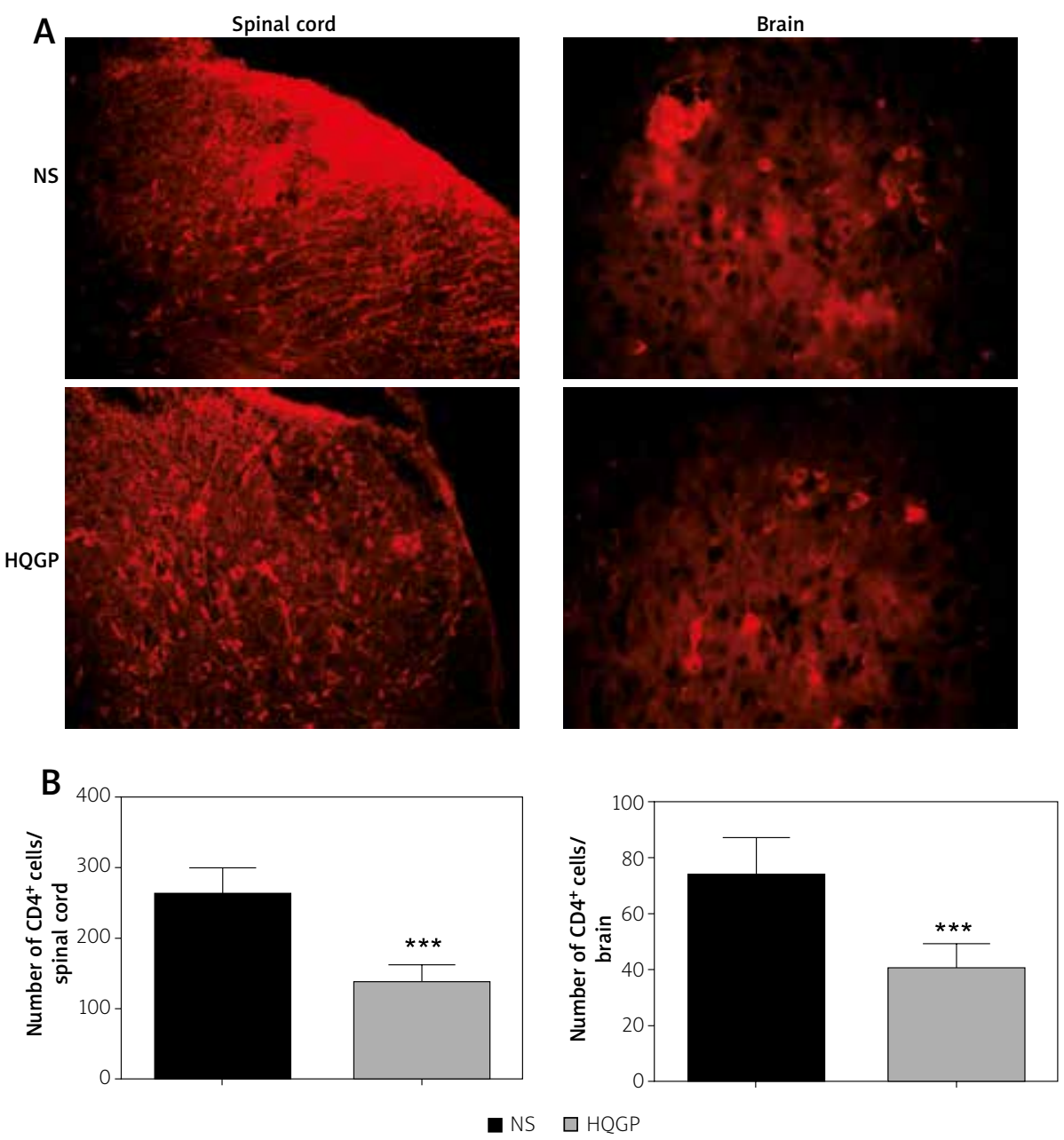

Fig. 5. Immunohistochemistry of $\mathrm{CD}^{+} \mathrm{T}$ cells in the CNS. The spinal cord and brain were harvested on day 18 p.i. and determined with anti-CD4 antibodies. A) Representative digital images (400x). B) The number of CD4 ${ }^{+}$cells was calculated by Image-Pro Plus 6.0 software. Results are expressed as mean \pm SD $(n=6$ each group). ${ }^{* * *} p<0.001$. One representative of three experiments with similar results is shown.

trating cells in the CNS. Our results showed that the expression of CCL2, CCL5 and CCR5 in HQGP-treated mice was significantly reduced compared with NS-treated control mice (Figs. 7, 8), suggesting that inhibition of expression of related chemokines/ chemokine receptors might reduce the infiltration of inflammatory cells into the CNS in EAE.

\section{Huangqi glycoprotein regulates the secretion of inflammatory cytokines in the periphery}

Infiltrating leukocytes in the CNS produce cytokines that promote an inflammatory cascade and attack myelin and neuronal axons. In this study, we measured levels of the cytokines TNF- $\alpha, \mathrm{IL}-6, \mathrm{IL}-1 \beta$ and IL-10 in the supernatant of cultured splenic MNCs by ELISA. We found that the levels of TNF- $\alpha$, IL- 6 and IL- $1 \beta$ were significantly reduced, while the levels of IL-10 were increased in mice treated with HQGP compared with EAE control mice (Fig. 9, $p<0.01, p<0.001$ ).

\section{Huangqi glycoprotein prevents axon damage and neuron loss in experimental autoimmune encephalomyelitis mice}

Microtubule related protein-2 (MAP-2) is used as a marker of neuronal axons and neuron nuclear antigen (NeuN) is a specific marker of mature neurons. 

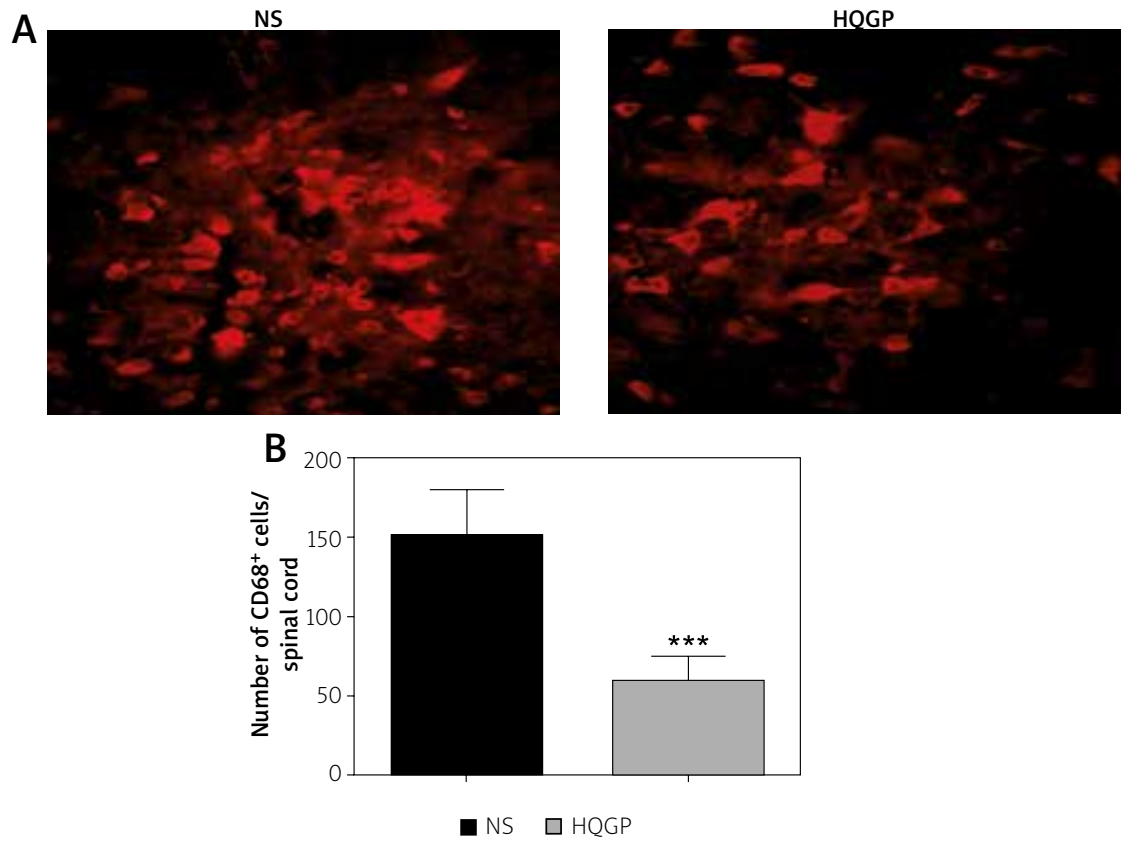

Fig. 6. Immunohistochemistry of $\mathrm{CD}^{+} 8^{+}$macrophages in the CNS. The spinal cord and brain were harvested on day 18 p.i. and determined with anti-CD68 antibodies. A) Representative digital images (400x). B) The number of $\mathrm{CD} 68^{+}$cells was calculated by Image-Pro Plus 6.0 software. Results are expressed as mean $\pm \mathrm{SD}$ ( $n=6$ each group). ${ }^{* * *} p<0.001$. One representative of three experiments with similar results is shown.

Given that both cell types are important for bona-fide function of the CNS and that EAE promotes a disturbance in these cells, we evaluated whether HQGP would protect mature neurons and neuronal axons from damage. Our results from immunofluorescence staining revealed that a dramatic axon damage and neuron loss in the spinal cord of NS-treated control mice was observed whereas HQGP increased MAP-2 and NeuN expression compared with NS-treated EAE control mice (Figs. 10, 11).

\section{Discussion}

Our present study showed that HQGP treatment effectively delayed disease onset and severity of EAE, inhibited CNS inflammatory infiltration, inhibited production of CCL2/CCL5 and proinflammatory cytokines, and protected neurons from inflammation-induced cell death. These results indicate that HQGP has anti-inflammatory and neuroprotective effects, and thus has potential as an alternative therapy for MS.

The migration of activated $T$ cells and macrophages in the CNS across the impaired blood brain barrier (BBB) plays an important role in the development of MS/EAE [3,27]. CD68 is the surface marker of macrophages and activated microglia [22]. CD11b, an important member of the leukocyte integrin $\beta 2$ subfamily of adhesion molecules, is transferred to the surface of monocytes and neutrophils under the stimulation of TNF- $\alpha, \mathrm{IL}-1$ and other cytokines. Monocytes and neutrophils then adhere to vascular endothelial cells and release of oxygen free radicals, protease and cytokines, leading to BBB damage and further immune cell infiltration in the CNS [5]. Our data indicate that HQGP can effectively block this pathogenic process, as shown by significantly reduced numbers of infiltrated $\mathrm{CD}^{+} \mathrm{T}$ cells, $\mathrm{CD}^{+} 8^{+}$ macrophages and $\mathrm{CD} 11 \mathrm{~b}^{+}$monocytes after HQGP treatment. Inhibiting the infiltration of immune cells into the CNS provides a solid basis for the effect of HQGP in reducing CNS tissue damage and EAE severity.

Myelin reactive immune response, which is initialized in the peripheral immune system, plays an important role in the pathogenesis of CNS autoimmunity. Peripheral immune organs contain a large 

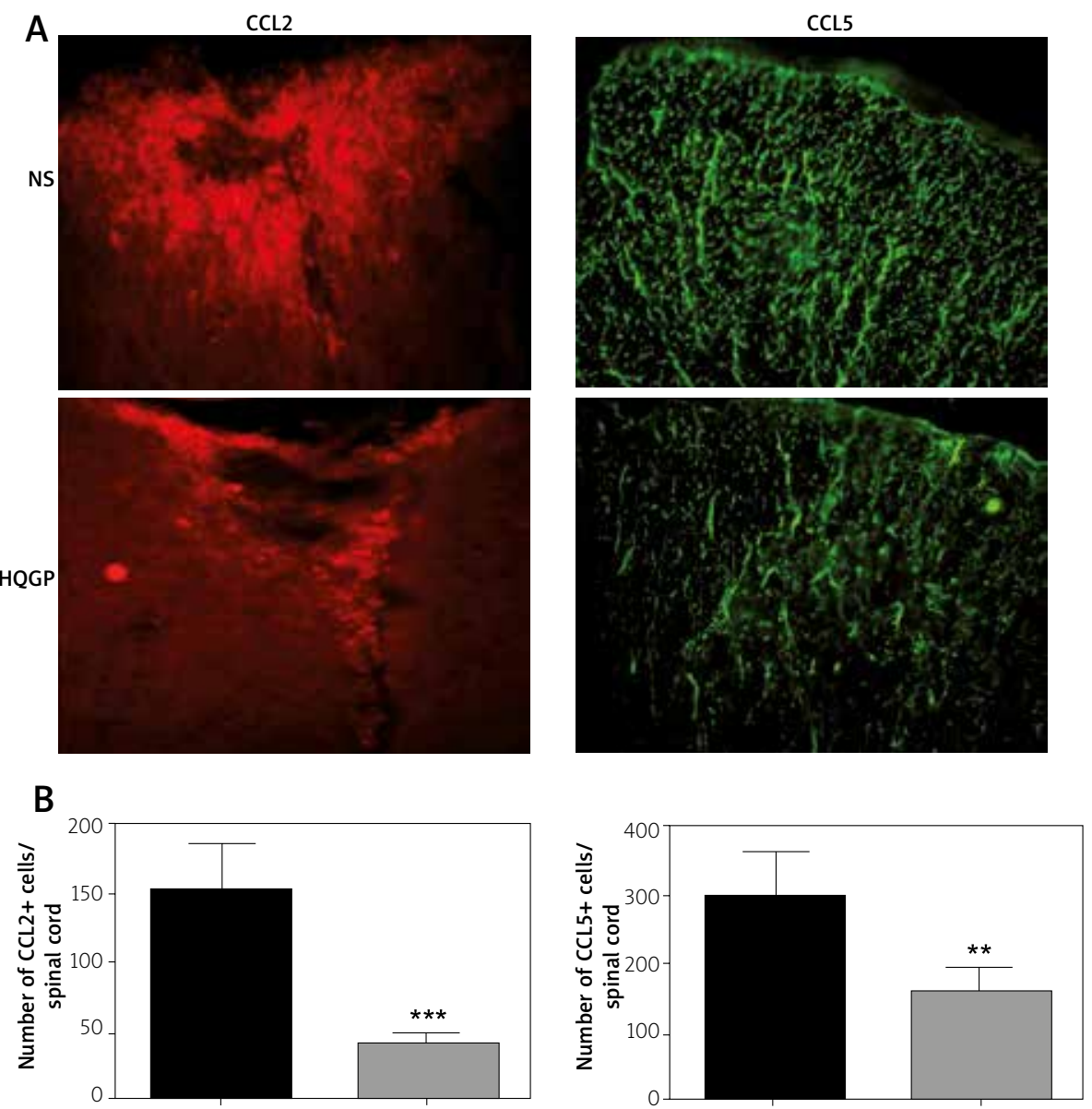

a $\mathrm{NS}$ HQGP

Fig. 7. Immunohistochemistry of $\mathrm{CCL}^{+}$and $\mathrm{CCL}^{+}$cells in the spinal cord. The spinal cord was harvested on day 18 p.i. and slices of $7 \mu \mathrm{m}$ were stained with anti-CCL2 and anti-CCL5 antibodies. A) Representative digital images (400x). B) The number of $\mathrm{CCL}^{+}$and $\mathrm{CCL}^{+}$cells was calculated by Image-Pro Plus 6.0 software. Results are expressed as mean \pm SD ( $n=6$ each group). ${ }^{* \star} p<0.01,{ }^{* \star *} p<0.001$. One representative of three experiments with similar results is shown.

number of lymphocytes and macrophages, which secrete several cytokines after inflammatory stimulation. TNF- $\alpha$, IL-1 $\beta$, and IL-6 are major inflammatory cytokines released by classically activated macrophages, while IL-10 produced by Th2 cells and alternatively activated macrophages is anti-inflammatory $[26,34]$. In our study, the supernatants of splenocytes contained significantly reduced levels of TNF- $\alpha, I L-1 \beta$ and IL- 6 in cultures from mice from the HQGP-treated group, while levels of IL-10 were upregulated. These results indicate that HQGP could effectively alleviate the peripheral immune inflam- matory response, and enhance an immunomodulatory response in EAE mice.

Activation of chemokine/chemokine receptor system can up-regulate the expression of integrin, activate leukocytes, and promote recruitment and degranulation of inflammatory cells. Among them, CCL5/CCR5 is of particular importance by mediating the infiltration of type 1 Th cells into the CNS during the development of EAE [6]. By combining with their receptor CCR5, CCL5 chemotactically accumulates monocytes and lymphocytes to enter the inflammatory region and participate in the inflammatory 

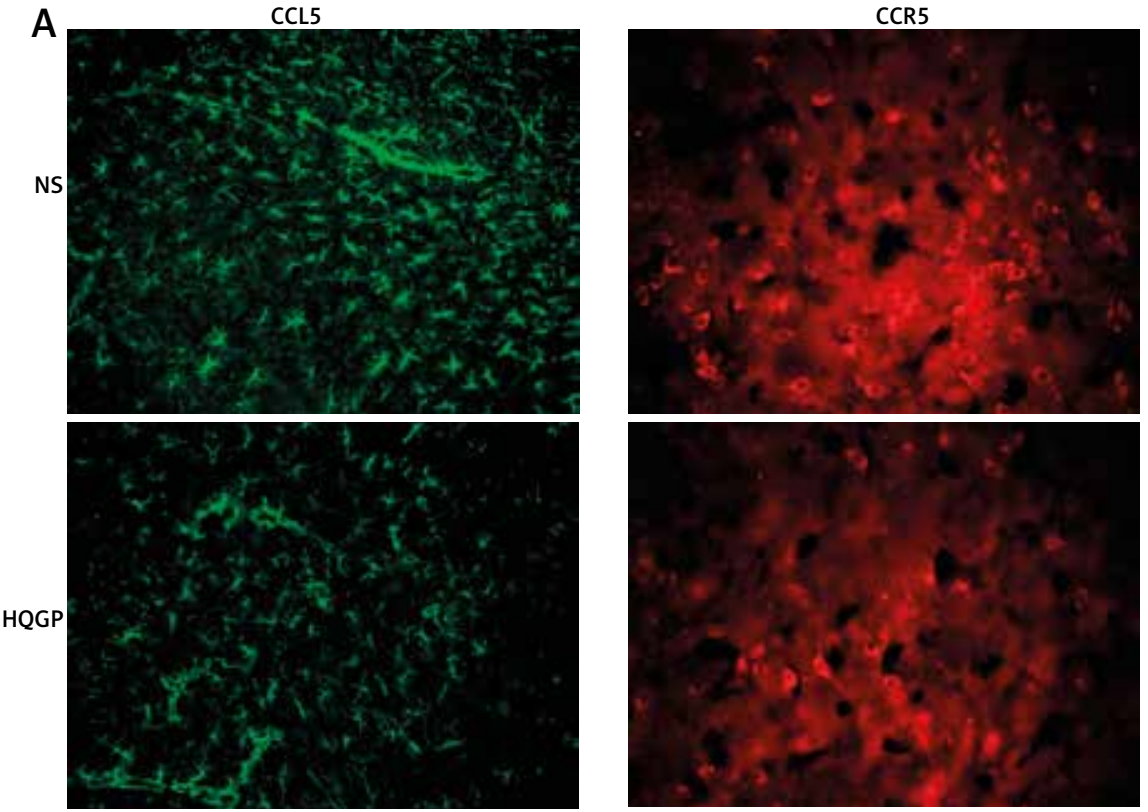

B
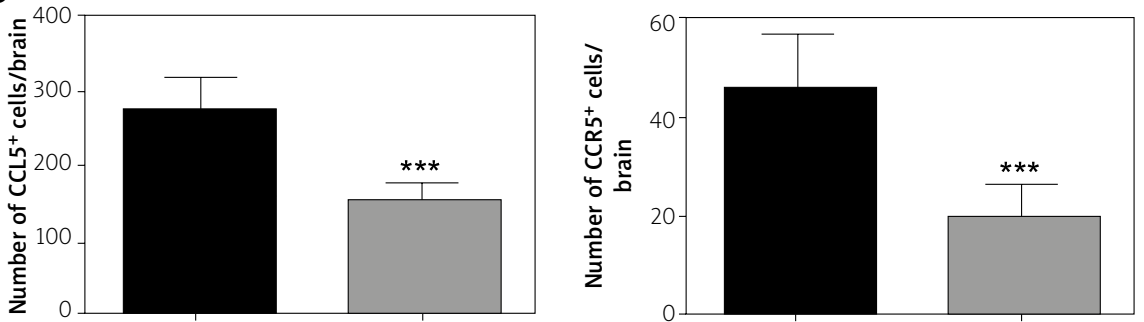

$\square$ NS $\square$ HQGP

Fig. 8. The expression of CCL5 and CCR5 in the brain. The brain was harvested on day 18 p.i. and slices of $7 \mu \mathrm{m}$ were stained with anti-CCL5 and anti-CCR5 antibodies. A) Representative digital images (400x). B) The number of $\mathrm{CCL}^{+}$and $\mathrm{CCR}^{+}$cells was calculated by Image-Pro Plus 6.0 software. Results are expressed as mean \pm SD $\left(n=6\right.$ each group). ${ }^{* * *} p<0.001$. One representative of three experiments with similar results is shown.

Fig. 9. The role of Huangqi glycoprotein (HQGP) in autoantigen-induced cytokine profile in EAE mice. EAE mice treated with HQGP or NS were sacrificed on day 18 p.i. Splenocytes were isolated, following stimulation with $\mathrm{MOG}_{35-55}$ $(25 \mu \mathrm{g} / \mathrm{ml})$ for $72 \mathrm{~h}$. Supernatants were harvested and analyzed for the levels of TNF- $\alpha$, IL- 6 , IL-1 $\beta$ and IL-10 using ELISA kits. Results are expressed as mean \pm SD ( $n=6$ each group). ${ }^{* *} p<0.01$, ${ }^{* * *} p<0.001$. One representative of two experiments with similar results is shown.

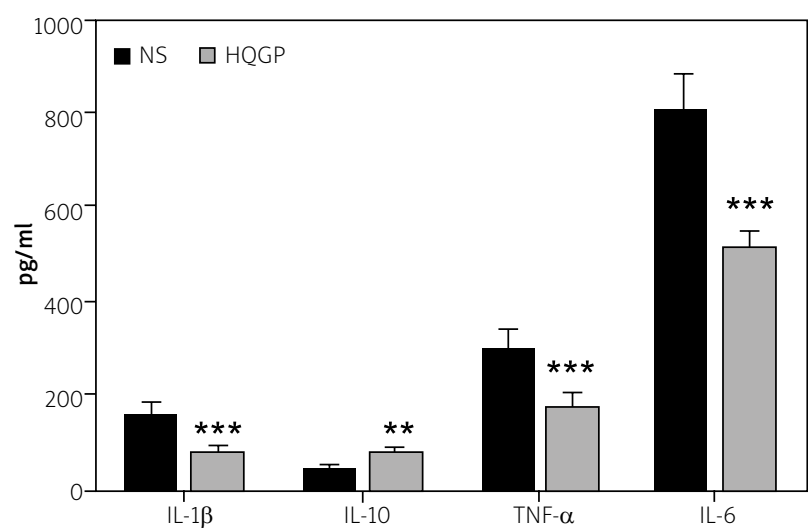



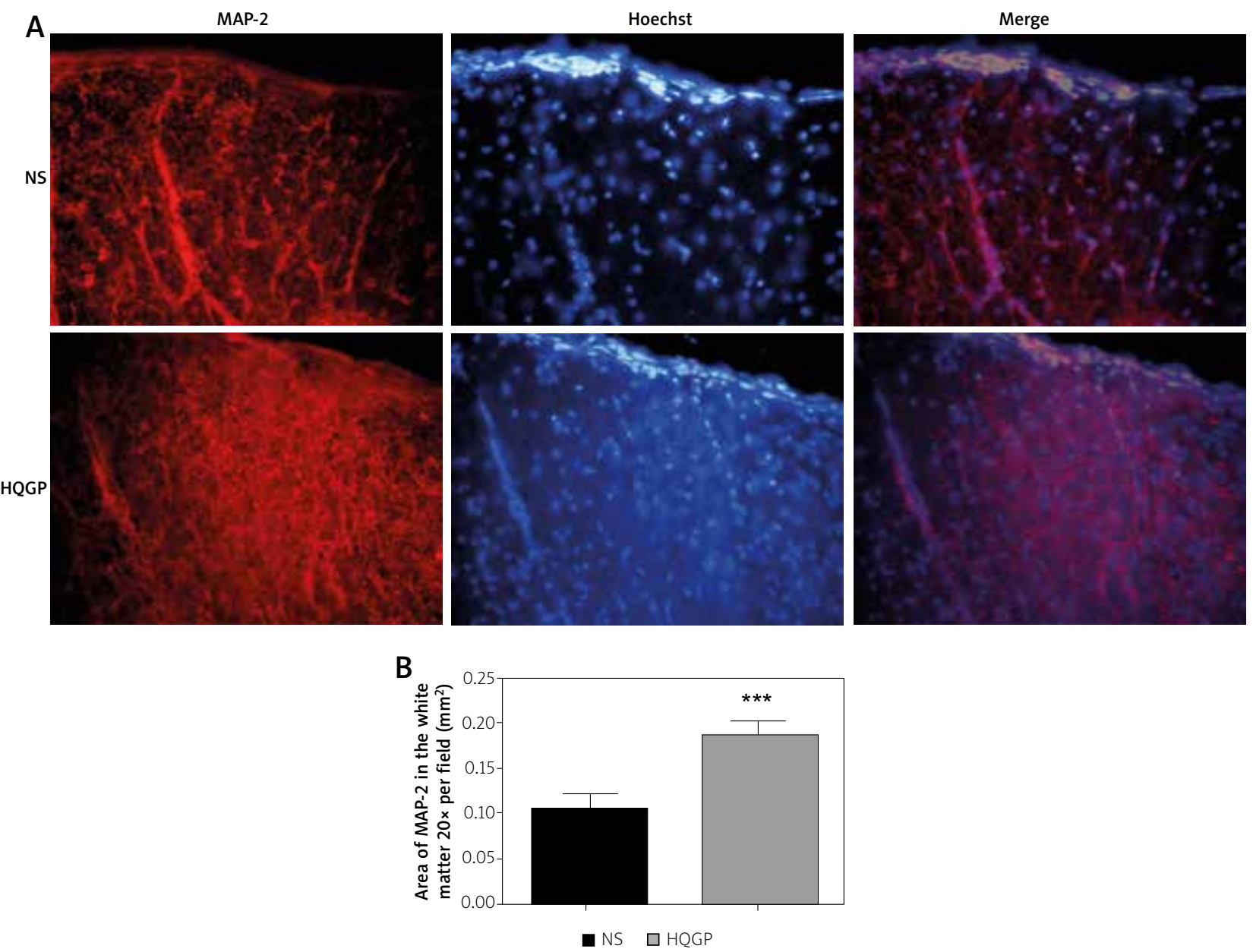

Fig. 10. Immunohistochemistry of MAP-2 in the spinal cord. The spinal cord was harvested on day 18 p.i. and slices of $7 \mu \mathrm{m}$ were stained with anti-MAP-2 antibodies. A) Representative digital images (400x). B) The number of MAP- $2^{+}$cells was calculated by Image-Pro Plus 6.0 software. Results are expressed as mean $\pm \mathrm{SD}$ ( $n=6$ each group). ${ }^{* * *} p<0.001$. One representative of three experiments with similar results is shown.

immune response. It was found that CCL5 was actively expressed in demyelinating plaques of MS, suggesting that it was involved in the formation of demyelination of MS [12]. CCL2 contributes to the recruitment of myeloid dendritic cells and macrophages during EAE [11], and reduction of CCL2 expression in the spinal cord could diminish infiltration of $\mathrm{CD}^{+} \mathrm{T}$ cells and inflammatory monocytes into the spinal cord [21]. Consistent with the above findings, HQGP treatment decreased the infiltration of inflammatory cells in the CNS, probably by significantly inhibiting the expression of CCL2, CCL5/CCR5 in EAE mice.

Microtubule-associated protein 2 is one of the main components of skeleton protein of nerve cells, which plays an important role in the development of neurons and axons. Reduced MAP-2 expression would lead to microtubules dysfunction such as transportation and plasticity in neurons [24]. It was reported that MAP-2 expression in the brain and spinal cord of EAE mice was significantly lower than that of the normal group [1]. NeuN is a stable and specific antigen marker of mature neurons, which reflects damage and repair of neurons. The positive expression intensity of NeuN could evaluate the maturity of neurons in this area $[16,29]$. In our study, we found that, while the expression of MAP-2 and NeuN was relatively low, HQCP treatment largely enhanced their expression. Thus, HQGP could inhibit axon loss and neuron injury induced by EAE and promote nerve repair. 
A
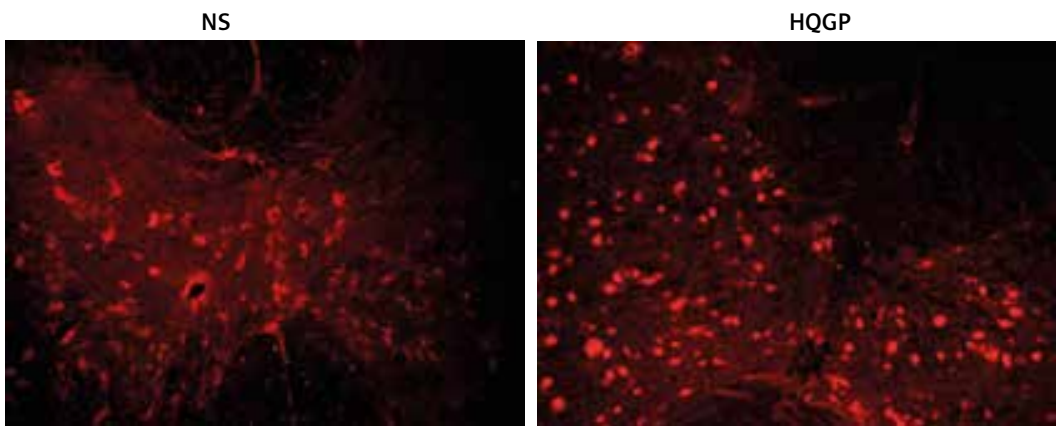

B

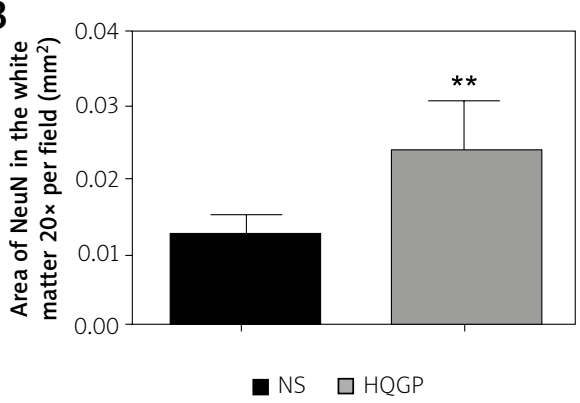

Fig. 11. Immunohistochemistry of NeuN expression in the spinal cord. The spinal cord was harvested on day 18 p.i. and slices of $7 \mu \mathrm{m}$ were stained with anti-NeuN antibodies. A) Representative digital images (200x). B) The number of $\mathrm{NeuN}^{+}$cells was calculated by Image-Pro Plus 6.0 software. Results are expressed as mean \pm SD ( $n=6$ each group). ${ }^{* *} p<0.01$. One representative of three experiments with similar results is shown.

In conclusion, the protective effect of HQGP on EAE mice is related to anti-inflammatory, immunoregulation and nerve tissue repair. HQGP, as an effective molecule derived from traditional herbal medicine, has potential to become a novel clinical therapy for MS.

\section{Acknowledgements}

Supported by: International S\&T Cooperation Program of China, No. 2013DFA30700; the National Natural Science Foundation of China, No. 81473577; the Natural Science Foundation of Shanxi Province, China, No. 2013011052-4; the Applied Basic Research Program of Datong, China, No. 2017135. We thank Dr. Guang-Xian Zhang for his critiques.

\section{Disclosure}

The authors report no conflict of interest.

\section{References}

1. An C, Wang YQ, Shi YM, Zhao H, Li M, Qi F, Zhang QX, Chen ZZ, Fan YP, Wang L. Effects of Bu Shen Yi Sui and its disassembled formulas on the expression of $\beta$-APP and MAP- 2 in the brain and spinal cord of mice with experimental autoimmune encephalomyelitis. J Emerg Tradit Chin Med 2016; 25: 565-568.
2. Bartosik-Psujek H, Stelmasiak Z. The levels of chemokines CXCL8, CCL2 and CCL5 in multiple sclerosis patients are linked to the activity of the disease. Eur I Neurol 2005; 12: 49-54.

3. Bennett J, Basivireddy J, Kollar A, Biron KE, Reickmann P, Jefferies WA, McQuaid S. Blood-brain barrier disruption and enhanced vascular permeability in the multiple sclerosis model EAE. J Neuroimmunol 2010; 229: 180-191.

4. Bogie JF, Stinissen P, Hendriks JJ. Macrophage subsets and microglia in multiple sclerosis. Acta Neuropathol 2014; 128: 191-213.

5. Bullard DC, Hu X, Schoeb TR, Axtell RC, Raman C, Barnum SR. Critical requirement of CD11b (Mac-1) on T cells and accessory cells for development of experimental autoimmune encephalomyelitis. J Immunol 2005, 175: 6327-6333.

6. Chen Q, Liu Y, Lu A, Ni K, Xiang Z, Wen K, Tu W. Influenza virus infection exacerbates experimental autoimmune encephalomyelitis disease by promoting type I T cells infiltration into central nervous system. J Autoimmun 2017; 77: 1-10.

7. Chiba K, Adachi K. Discovery of fingolimod, the sphingosine 1-phosphate receptor modulator and its application for the therapy of multiple sclerosis. Future Med Chem 2012; 4: 771-781.

8. Constantinescu CS, Farooqi N, O'Brien K, Gran B. Experimental autoimmune encephalomyelitis (EAE) as a model for multiple sclerosis (MS). Br J Pharmacol 2011; 164: 1079-1106.

9. Dargahi N, Katsara M, Tselios T, Androutsou ME, de Courten M, Matsoukas J, Apostolopoulos V. Multiple sclerosis: immunopathology and treatment update. Brain Sci 2017; 7: E78.

10. Dendrou CA, Fugger L, Friese MA. Immunopathology of multiple sclerosis. Nat Rev Immunol 2015; 15: 545-558. 
11. Dogan RN, Elhofy A, Karpus WJ. Production of CCL2 by central nervous system cells regulates development of murine experimental autoimmune encephalomyelitis through the recruitment of TNF- and iNOS-expressing macrophages and myeloid dendritic cells. J Immunol 2008; 180: 7376-7384.

12. Dos Santos AC, Roffê E, Arantes RM, Juliano L, Pesquero IL, Pesquero JB, Bader M, Teixeira MM, Carvalho-Tavares J. Kinin B2 receptor regulates chemokines CCL2 and CCL5 expression and modulates leukocyte recruitment and pathology in experimental autoimmune encephalomyelitis (EAE) in mice. J Neuroinflammation 2008; 5: 49.

13. Jiang JB, Qiu JD, Yang LH, He JP, Smith GW, Li HQ. Therapeutic effects of astragalus polysaccharides on inflammation and synovial apoptosis in rats with adjuvant-induced arthritis. Int J Rheum Dis 2010; 13: 396-405.

14. Kan QC, Zhang HJ, Zhang Y, Li X, Xu YM, Thome R, Zhang ML, Liu N, Chu YJ, Zhang GX, Zhu L. Matrine treatment blocks NogoAinduced neural inhibitory signaling pathway in ongoing experimental autoimmune encephalomyelitis. Mol Neurobiol 2017; 54: 8404-8418

15. Kim J, Moon E, Kwon S. Effect of Astragalus membranaceus extract on diabetic nephropathy. Endocrinol Diabetes Metab Case Rep 2014; 2014: 140063.

16. Kim KK, Nam J, Mukouyama YS, Kawamoto S. Rbfox3-regulated alternative splicing of Numb promotes neuronal differentiation during development. J Cell Biol 2013; 200: 443-458.

17. Kim RY, Hoffman AS, Itoh N, Ao Y, Spence R, Sofroniew MV, Voskuhl RR. Astrocyte CCL2 sustains immune cell infiltration in chronic experimental autoimmune encephalomyelitis. J Neuroimmunol 2014; 274: 53-61.

18. Legroux L, Arbour N. Multiple sclerosis and T lymphocytes: an entangled story. J Neuroimmune Pharmacol 2015; 10: 528-546.

19. Mahad D, Callahan MK, Williams KA, Ubogu EE, Kivisäkk P, Tucky B, Kidd G, Kingsbury GA, Chang A, Fox RJ, Mack M, Sniderman MB, Ravid R, Staugaitis SM, Stins MF, Ransohoff RM. Modulating CCR2 and CCL2 at the blood-brain barrier: relevance for multiple sclerosis pathogenesis. Brain 2006; 129: 212-223.

20. Mondal S, Pahan K. Cinnamon ameliorates experimental allergic encephalomyelitis in mice via regulatory T cells: implications for multiple sclerosis therapy. PLoS One 2015; 10: e0116566.

21. Nacka-Aleksić M, Stojić-Vukanić Z, Pilipović I, Vujnović I, Bufan B, Dimitrijević M, Leposavić G. Strain specificities in cellular and molecular immunopathogenic mechanisms underlying development of experimental autoimmune encephalomyelitis in aged rats. Mech Ageing Dev 2017; 164: 146-163.

22. Peferoen LA, Vogel DY, Ummenthum K, Breur M, Heijnen PD, Gerritsen WH, Peferoen-Baert RM, van der Valk P, Dijkstra CD, Amor S. Activation Status of human microglia is dependent on lesion formation stage and remyelination in multiple sclerosis. J Neuropathol Exp Neurol 2015; 74: 48-63.

23. Pittaluga A. CCL5-glutamate cross-talk in astrocyte-neuron communication in multiple sclerosis. Front Immunol 2017; 8: 1079.

24. Prieto-Gómez B, Velázquez-Paniagua M, Cisneros LO, ReyesVázquez C, Jiménez-Trejo F, Reyes ME, Mendoza-Torreblanca J, Gutiérrez-Ospina G. Melatonin attenuates the decrement of dendritic protein MAP-2 immuno-staining in the hippocampal
CA1 and CA3 fields of the aging male rat. Neurosci Lett 2008; 448: 56-61.

25. Seehusen F, Baumgärtner W. Axonal pathology and loss precede demyelination and accompany chronic lesions in a spontaneously occurring animal model of multiple sclerosis. Brain Pathol 2010; 20: 551-559.

26. Sharma R, Tiku AB. Emodin inhibits splenocyte proliferation and inflammation by modulating cytokine responses in a mouse model system. J Immunotoxicol 2016; 13: 20-26.

27. Sonar SA, Shaikh S, Joshi N, Atre AN, Lal G. IFN- $\gamma$ promotes transendothelial migration of CD4+ T cells across the blood-brain barrier. Immunol Cell Biol 2017; 95: 843-853.

28. Szczuciński A, Losy J. Chemokines and chemokine receptors in multiple sclerosis. Potential targets for new therapies. Acta Neurol Scand 2007; 115: 137-146.

29. Toyoshima Y, Sekiguchi S, Negishi T, Nakamura S, Ihara T, Ishii Y, Kyuwa S, Yoshikawa Y, Takahashi K. Differentiation of neural cells in the fetal cerebral cortex of cynomolgus monkeys (Macaca fascicularis). Comp Med 2012; 62: 53-60.

30. Vogt J, Paul F, Aktas O, Müller-Wielsch K, Dörr J, Dörr S, Bharathi BS, Glumm R, Schmitz C, Steinbusch H, Raine CS, Tsokos M, Nitsch R, Zipp F. Lower motor neuron loss in multiple sclerosis and experimental autoimmune encephalomyelitis. Ann Neurol 2009; 66: 310-322.

31. Wu J, Liu DF, Chen Y, Yan JQ, Xiao J, Li Y. Effects of Radix Astragali on IL-1 $\beta$, TNF- $\alpha$, and antigen expression of peripheral blood mononuclear cells in patients with Graves disease. Chin J Integr Trad West Med 2011; 31: 1487-1490.

32. Zhang H, Guo M, Zhang L, Xue H, Chai Z, Yan Y, Xing Y, Xiao B, Zhang P, Ma C. Anti-inflammatory effect and mechanisms of Huangqi glycoprotein in treating experimental autoimmune encephalomyelitis. Folia Neuropathol 2017; 55: 308-316.

33. Zhang P, Guo M, Xing Y, Yang D, Zhang L, Li Y, Xue H, Xiao B, Ma C. Immunomodulatory effect of Huangqi glycoprotein on mice with experimental autoimmune encephalomyelitis. Chin J Cell Mol Imm 2016; 32: 54-58.

34. Zhou H, Wang Y, Lian Q, Yang B, Ma Y, Wu X, Sun S, Liu Y, Sun B. Differential IL-10 production by DCS determines the distinct adjuvant effects of LPS and PTX in EAE induction. Eur I Immunol 2014; 44: 1352-1362. 